\title{
Polarization Reconfigurable Microstrip Antennas For Wireless Communication
}

\author{
A. Bharathi ${ }^{1}$ and P.Kavitha ${ }^{2}$ \\ ${ }^{1}$ CVR College of Engineering, Department of ECE., Ibrahimpatan, R.R.District, A.P., India \\ Email: bharathig8@gmail.com \\ ${ }^{2}$ CVR College of Engineering, Department of ECE., Ibrahimpatan, R.R.District, A.P., India \\ Email: kavitha.ou@gmail.com
}

\begin{abstract}
Antenna systems that utilize polarization diversity are gaining popularity due to development of wireless communication in recent years. This paper presents a novel polarization reconfigurable antenna to improve the reliability of wireless communication system. The structure is very simple obtained by trimming the corners of square patch fed with microstrip line feed, and adding four small triangular shape conductors at the corners. Introducing independently biased pin diodes into the gap between patch and triangular conductors, the antenna can radiate linear polarization (LP), or left hand circular polarization (LHCP) or Right hand circular polarization (RHCP). The proposed antenna designed for $1.6 \mathrm{GHz}$ range, achieve excellent performance with $\mathrm{LP}$, RHCP, LHCP at the same frequency and are built on FR4Epoxy substrate. The antenna architecture is interesting for commercial wireless applications because it is compatible with modern fabrication processes and can be realized on low cost dielectric materials.
\end{abstract}

Index Terms - Microstrip antenna, Polarization.

\section{INTRODUCTION}

Microstrip antennas are widely used in Wireless and mobile communication systems applications due to their low profile, light weight, conformable with RF circuitry and easy fabrication [1]. They are usually designed for singlemode operation that radiates mainly linear polarization. In some applications, such as satellite communications, however, a circularly polarized system is more suitable because of its insensitivity to transmitter and receiver orientations [2]. In this regard, integrated systems with different communication networks have attracted significant attention. To achieve such multi-operation, numerous researchers have investigated microstrip antennas with switchable polarization. The switchable property allows the user to roam any existing network and have only a single handset to access a great number of services. Therefore, they can be utilized to realize frequency reuse [3]. Polarization diversity of reception is important to counter the effects of fading in communication, especially in mobile communication [4]. In addition, a microstrip antenna with switchable polarization is very important because many current communication and sensor systems require a high degree of polarization control to optimize system performance [5]. Practical applications of this technique have been described in [3], [4], and [6]. Based on the same concept, a novel reconfigurable microstrip antenna allowing polarization switching is proposed and carefully examined in this study. Individually biased PIN diodes introduced into the corners of antennas enable independent control of polarization sense.

\section{ReCONFIGURABLE Microstrip PATCh ANTENNA DESIGN}

A. Polarization Reconfigurable Patch Antenna Design:

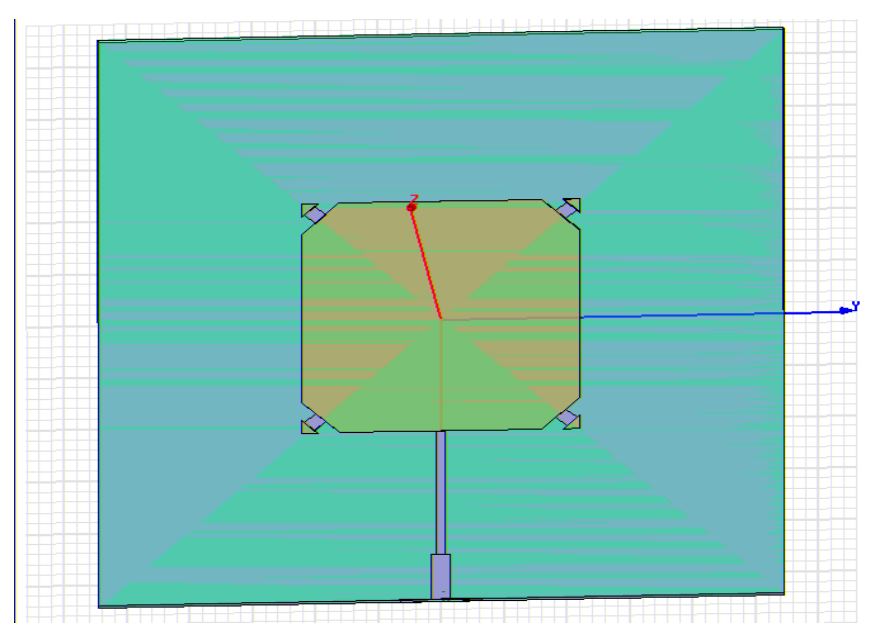

Figure 1. Configuration of the patch antenna with polarization diversity.

Fig. 1 shows the proposed Microstrip antenna with square patch having a side length of $44.3 \mathrm{~mm}$ printed on a substrate with a relative dielectric constant of 4.4 and thickness of 1.6 $\mathrm{mm}$. Its resonant frequency is $1.6 \mathrm{GHz}$.

The truncated corners have an equal side length $6 \mathrm{~mm}$ and are used to provide perturbation, so as to obtain two orthogonal near-degenerate resonant modes for circular polarization. The technique of truncating corners of a square patch to obtain single-feed circular polarization operation is well known and has been widely used in practical designs [7]. This corner-truncated square patch has four small parasitic conductors of triangular shape with a side length of $2.3 \mathrm{~mm}$. As the size of the PIN diode is $2.5 \times 1.4 \mathrm{~mm}$, the gap between the square patch and triangular conductors is designated as $1.4 \mathrm{~mm}$. The impedance and radiation properties of reconfigurable patch antenna is investigated numerically and experimentally. Simulations are carried out using HFSS, a commercial EM Simulator using Finite Element Method (FEM). The proposed antenna shows excellent radiation characteristics. The return loss, the gain, and the radiation patterns of the proposed antenna have been simulated and measured under different biasing conditions 
of the diodes. Different polarization is achieved by changing the geometry of antenna according to on/off state of diodes. Figure shows the configuration of the patch antenna which radiates linearly polarized waves.

TABLE I:

\begin{tabular}{|l|l|l|l|l|l|}
\hline & Diode1 & Diode2 & Diode3 & Diode4 & polarization \\
\hline A1 & ON & ON & ON & ON & LP \\
\hline A2 & ON & OFF & ON & OFF & LHCP \\
\hline A3 & OFF & ON & OFF & ON & RHCP \\
\hline
\end{tabular}

The resonant frequency remains same as $1.6 \mathrm{GHz}$ for all the three polarizations (LP, RHCP, LHCP). The diode configurations are shown in Table I. for different states of polarization.

Fig. 2 shows the return loss and axial ratio plots for LP state when all the four diodes are on. A $-10 \mathrm{~dB}$ return loss is obtained at resonant frequency with an Axial Ratio of 41 $\mathrm{dB}$.
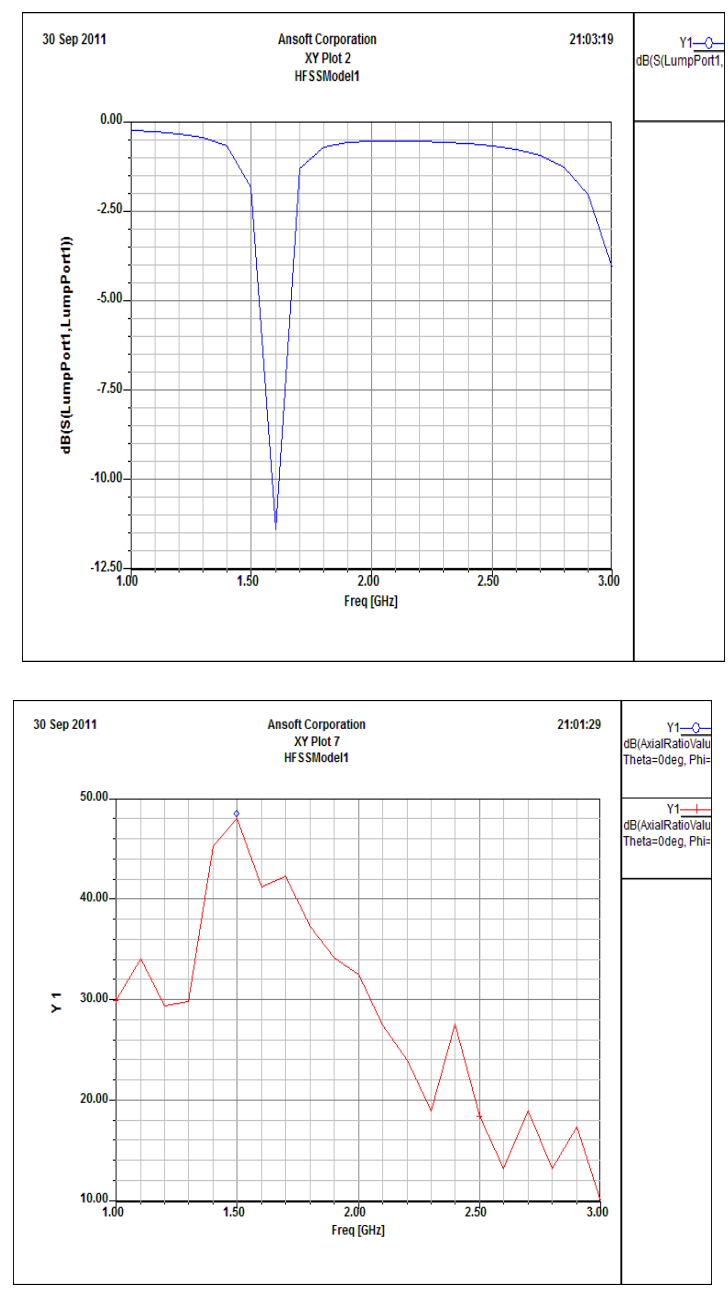

Figure 2 Simulated results for (a) Return loss and (b) Axial ratio giving LP when all the diodes are on.

Fig. 3 shows the return loss and axial ratio plots for LHCP when diode 1 and diode 3 are on. A $-10 \mathrm{~dB}$ return loss is obtained at resonant frequency with an Axial Ratio of 1.5 $\mathrm{dB}$.
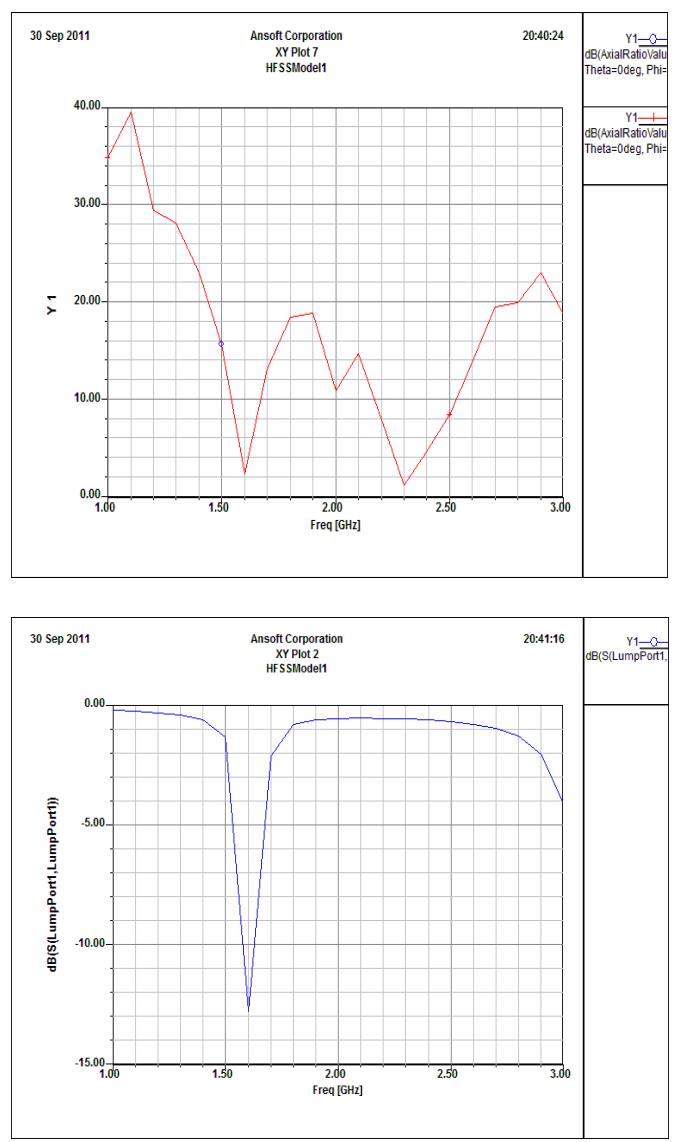

Figure 3. Simulated results for (a) Return loss and (b) Axial ratio giving $\mathrm{CP}$ when diode 1 and diode 3 are on.
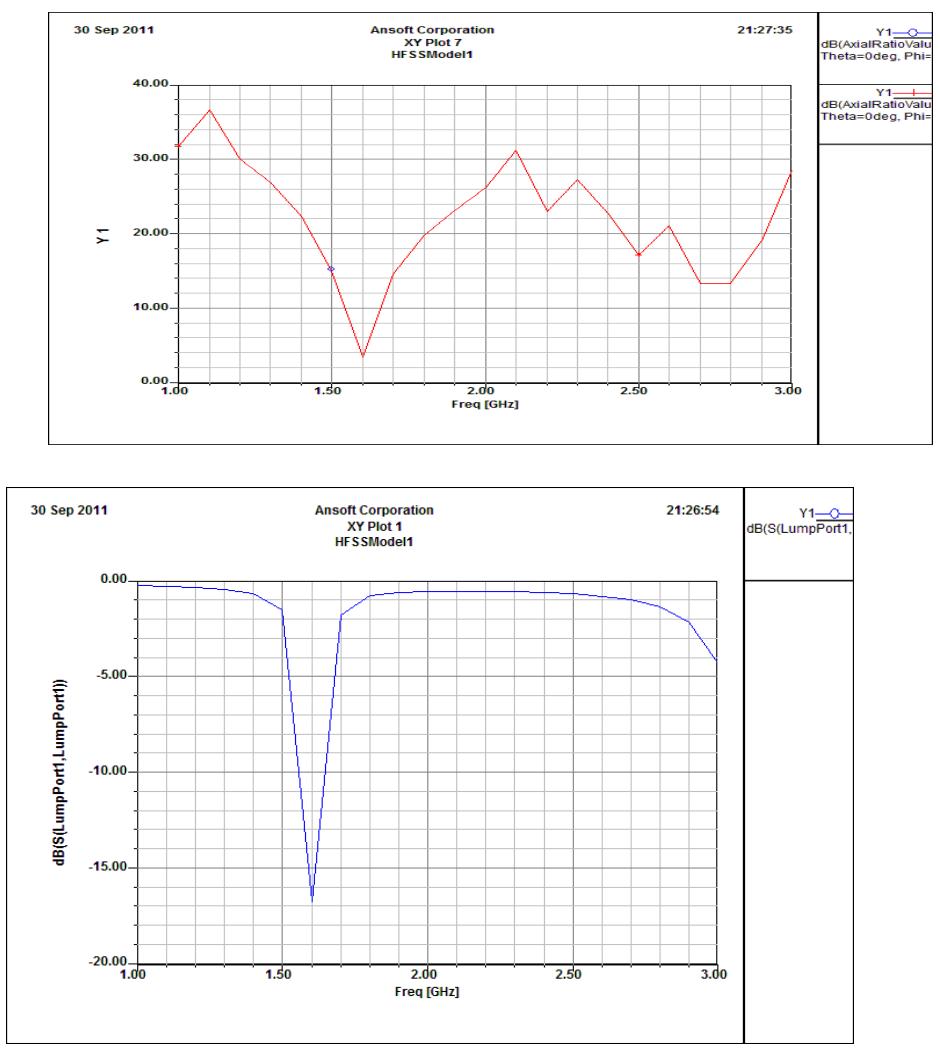

Figure 4. Simulated results for (a) Return loss and (b) Axial ratio giving $\mathrm{CP}$ when diode 2 and diode 4 are on. 
Fig. 4 shows the return loss and axial ratio plots for RHCP when diode 2 and diode 4 are on. A -10d B return loss is obtained at resonant frequency with an Axial Ratio of 2 $\mathrm{dB}$.

\section{CONCLUSIONS}

A Microstrip antenna with switchable polarization at $1.6 \mathrm{GHz}$ is presented. It shows different polarizations achieved by using pin diodes. It has uniplanar design which allows integration of active/passive elements. It can be used in applications where polarization diversity is required. Without any change in feed mechanism the antenna can radiate $\mathrm{LP}, \mathrm{RHCP}$ and $\mathrm{LHCP}$ by controlling bias voltage. This design has desirable features for wireless communication applications such as WLAN, Satellite Links and Space Robots.

\section{REFERENCES}

[1] J. R. James, P. S. Hall, and C. Wood, Microstrip Antenna Theory and Design. London, U.K.: Peter Peregrinus, 1981.

[2] F.-S. Chang, K.-L. Wong, and T.-W. Chiou, "Low-cost broadband circularly polarized patch antenna," IEEE Trans. Antennas Propagat., vol. 51, pp. 3006-3009, Oct. 2003.

[3] F. Yang and Y. Rahmat-Samii, "A reconfigurable patch antenna using switchable slots for circular polarization diversity," IEEE Microwave Wireless Compon. Lett., vol. 12, pp. 96-98, Mar. 2002.

[4] M. K. Fries, M. Grani, and R. Vahldieck, "A reconfigurable slot antenna with switchable polarization," IEEE MicrowaveWireless Compon. Lett., vol. 13, pp. 490-492, Nov. 2003.

[5] K. C. Gupta and P. S. Hall, Analysis and Design of Integrated Circuit Antenna Modules. New York: Wiley, 2000.

[6] P. M. Haskins, P. S. Hall, and J. S. Dahele, "Polarization-agile active patch antenna," Electron. Lett., vol. 30, pp. 98-99, Jan. 1994.

[7] W.-S. Chen, C.-K. Wu, and K.-L. Wong, "Novel compact circularly polarized square microstrip antenna," IEEE Trans. Antennas Propagat., vol. 49, pp. 340-342, Mar. 2001. 